\title{
Erratum to: Nonsingular dislocation and crack fields: implications to small volumes
}

\author{
J. Kioseoglou • I. Konstantopoulos • \\ G. Ribarik · G. P. Dimitrakopulos · \\ E. C. Aifantis
}

Published online: 16 September 2009

(C) Springer-Verlag 2009

Erratum to: Microsyst Technol (2009) 15:117-121

DOI 10.1007/s00542-008-0700-6

- In the legend of Fig. 5 the symbol $K$ for the intensity should not be confused with the $K$ used to designate Bessel functions in Eqs. 5 and 8 and the third line in this figure caption should read

“. ..K $-K_{0}$, where $K=2 \sin \theta / \lambda$ and $K_{0}$ is the value of $K$ at the exact..."

- The factor $b$ in the first term of the r.h.s. of Eq. 8 should be replaced by by; i.e. Eq. 8 should read

$$
\begin{aligned}
\varepsilon_{x}= & -\frac{b}{4 \pi(1-v)} y\left[\frac{(1-2 v) r^{2}+2 x^{2}}{r^{4}}\right] \\
& +\frac{b}{2 \pi(1-v)} y\left[\left(y^{2}-v r^{2}\right) \Phi_{1}+\left(3 x^{2}-y^{2}\right) \Phi_{2}\right]
\end{aligned}
$$

The online version of the original article can be found under doi:10.1007/s00542-008-0700-6.

J. Kioseoglou · G. P. Dimitrakopulos

Department of Physics, Aristotle University of Thessaloniki,

54124 Thessaloníki, Greece

I. Konstantopoulos · G. Ribarik · E. C. Aifantis

Laboratory of Mechanics and Materials,

Aristotle University of Thessaloniki, 54124 Thessaloníki, Greece

G. Ribarik

Department of Materials Physics, Eötvös University Budapest,

POB 32, 1518 Budapest, Hungary

E. C. Aifantis $(\square)$

Center for the Mechanics of Material Instabilities

and Material Processes, Michigan Technological University,

Houghton, MI 49931, USA

e-mail: mom@mom.gen.auth.gr 